zation of FGF23 as a causative factor of tumorinduced osteomalacia. Proc. Natl. Acad. Sci.U.S. A. 98:6500-6505.

14. Shimada, T., et al. 2002. Mutant FGF-23 responsible for autosomal dominant hypophosphatemic rickets is resistant to proteolytic cleavage and causes hypophosphatemia in vivo. Endocrinology. 143:3179-3182.

15. Yamashita, T., Konishi, M., Miyake, A., Inui, K., and Itoh, N. 2002. Fibroblast growth factor (FGF)-23 inhibits renal phosphate reabsorption by activation of the mitogen-activated protein kinase pathway. J. Biol. Chem. 277:28265-28270.

16. Shimada, T., et al. 2002. Targeted ablation of FGF-23 causes hyperphosphatemia, increased 1,25-dyidrxyvitam D levels and severe growth retardation. J. Bone Miner. Res. 17:S168. (Abstr.)

17. Liu, S., et al. 2003. Regulation of FGF23 expression but not degradation by Phex. J. Biol. Chem. 10.1074/jbc.M304544200.

18. Holm, I.A., Huang, X., and Kunkel, L.M. 1997. Mutational analysis of the PHEX gene in patients with X-linked hypophosphatemic rickets. Am. J. Hum. Genet. 60:790-797.

19. Weber, T.J., Liu, S., Indirdason, O.S., and Quarles, L.D. 2003. Serum FGF23 levels in normal and disordered phosphorus homeostasis. J. Bone Miner. Res. 18:1227-1234.

20. Bowe, A.E., et al. 2001. FGF-23 inhibits renal tubular phosphate transport and is a PHEX substrate. Biochem. Biophys. Res. Commun. 284:977-981.

21. Jonsson, K.B., et al. 2003. Fibroblast growth factor 23 in oncogenic osteomalacia and X-linked hypophosphatemia. N. Engl.J. Med. 348:1656-1663

22. Yamazaki, Y., et al. 2002. Increased circulatory level of biologically active full-length FGF-23 inpatients with hypophosphatemic rickets/osteomalacia. J. Clin. Endocrinol. Metab. 87:4957-4960.

23. Riminucci, M., et al. 2003. FGF-23 in fibrous dysplasia of bone and its relationship to renal phosphate wasting. J. Clin. Invest. 112:683-692. doi:10.1172/JCI200318399.

24. Gowen, L.C., et al. 2003. Targeted disruption of the osteoblast/osteocyte factor 45 gene (OF45) results in increased bone formation and bone mass. J. Biol. Chem. 278:1998-2007.

25. Petersen, D.N., Tkalcevic, G.T., Mansolf, A.L., Rivera-Gonzalez, R., and Brown, T.A. 2000. Identification of osteoblast/osteocyte factor 45 (OF45), a bone-specific cDNA encoding an RGD-containing protein that is highly expressed in osteoblasts and osteocytes. J. Biol. Chem. 275:36172-36180.

26. Qin, C., et al. 2003. Evidence for the proteolytic processing of dentin matrix protein 1: identification and characterization of processed fragments and cleavage sites. J. Biol. Chem. 10.1074/jbc.M305315200.

27. Liu, S., Brown, T.A., Xiao, Z., Guo, R., and Quarles, L.D. 2003. Deletion of Mepe in Hyp mice fails to correct hypophosphatemia but partially rescues abnormal mineralization ex vivo. J. Bone Miner. Res. In press.

28. Guo, R., Rowe, P.S.N., Liu, S., and Quarles, L.D 2002. Inhibition of MEPE cleavage by Phex. Biochem. Biophys. Res. Commun. 297:38-45.
29. Berndt, T., et al. 2003. Secreted frizzled-related protein 4 is a potent tumor-derived phosphaturic agent. J. Clin. Invest. 112:785-794. doi:10.1172/ JCI200318563

30. Kumar, R. 2002. New insights into phosphate homeostasis: fibroblast growth factor 23 and frizzled-related protein- 4 are phosphaturic factors derived from tumors associated with osteomalacia. Curr. Opin. Nephrol. Hypertens. 11:547-553.

31. Nesbitt, T., et al. 1999. Coordinated maturational regulation of PHEX and renal phosphate transport inhibitory activity: evidence for the pathophysiological role of PHEX in X-linked hypophosphatemia. J. Bone Miner. Res. 14:2027-2035.

32. Beck, L., et al. 1998. Targeted inactivation of $\mathrm{Npt} 2$ in mice leads to severe renal phosphate wasting, hypercalciuria, and skeletal abnormalities. Proc. Natl. Acad. Sci. U. S. A. 95:5372-5377.

33. Xiao, Z.S., et al. 1998. Intrinsic mineralization defect in Hyp mouse osteoblasts. Am. J. Physiol. 275:E700-E708

34. Boyden, L.M., et al. 2002. High bone density due to a mutation in LDL-receptor-related protein 5. N. Engl. J. Med. 346:1513-1521.

35. Little, R.D., et al. 2002. A mutation in the LDL receptor-related protein 5 gene results in the autosomal dominant high-bone-mass trait. Am. J. Hum. Genet. 70:11-19.

36. Kato, M., et al. 2002. Cbfa1-independent decrease in osteoblast proliferation, osteopenia, and persistent embryonic eye vascularization in mice deficient in Lrp5, a Wnt coreceptor. J. Cell Biol. 157:303-314.

\title{
Come forth CD1d: Hsp110 in the regulation of intestinal epithelial CD1d expression
}

\author{
Christopher V. Nicchitta
}

Department of Cell Biology, Duke University Medical Center, Durham, North Carolina, USA

\begin{abstract}
CD1d, a nonclassical MHC class I-like molecule, is prominently expressed on intestinal epithelial cells and is thought to function in the regulation of intestinal intraepithelial lymphocyte activity. Hsp110, an abundant heat shock protein present in essentially all mammalian tissues, has now been shown to upregulate CD1d expression in colonic tissue culture cell lines (see the related article beginning on page 745). Might this abundant chaperone serve an autocrine function in the regulation of CD1d expression?
\end{abstract}

J. Clin. Invest. 112:646-648 (2003). doi:10.1172/JCI200319614.

Intestinal epithelial cells (IECs) serve as the cellular barrier between the enormously challenging antigenic

Address correspondence to: Christopher V.
Nicchitta, Department of Cell Biology,
Duke University Medical Center, Durham,
North Carolina 27710, USA.
Phone: (919) 684-8948; Fax: (919) 684-5481;
E-mail: c.nicchitta@cellbio.duke.edu.
Conflict of interest: The author has declared
that no conflict of interest exists.
Nonstandard abbreviations used: intestinal
epithelial cell (IEC); inflammatory bowel
disease (IBD); heat shock protein 110 (Hsp110). environment of the intestinal lumen consisting of dietary antigens, viruses, bacteria, and fungi - and the mucosal immune system, the largest lymphoid tissue of the body. Given this remarkable immunological challenge, it comes as no surprise that both normal and pathological intestinal epithelial physiology involves complex interactions among the immune and nonimmune cells of the gut, the intestinal flora, and the activities of the sympathetic, parasympathetic, and enteric nervous systems. Unfortunately, such complex interactions often go awry, resulting in the chronic, $\mathrm{T}$ lymphocyte-mediated mucosal injury that characterizes inflammatory bowel diseases (IBDs).

The molecular etiology of IBDs is under continuing investigation, with current views emphasizing interactions between environmental pathogens and an altered, or genetically permissive, immune system (1). Recent studies have implicated CD1d in the regulation of intestinal inflammation, though here again, the precise immunological mechanism for such regulation remains to be identified (2-4). CD1d is a nonclassical MHC molecule that functions in the development of NK1.1 $1^{+} \mathrm{T}$ cells and has been implicated in the process of IEC-elicited $\mathrm{T}$ cell proliferation $(5,6)$. Interestingly, antibody cross-linking of CD1d has been demonstrated to elicit epithelial IL-10 production (7). IL-10 is known to serve important functions in the regulation of mucosal inflammation, and, importantly, IL-10-deficient mice coincidentally develop IBD (reviewed in ref. 8). From these observations has come the intriguing suggestion that ligation of CD1d may serve to suppress mucosal inflamma- 
tion, perhaps via activation of autocrine, IL-10-dependent responses in IECs (7). Such observations point to a critical need to understand the regulation of CD1d expression, CD1d recognition, the antigen(s) presented by IEC CD1d, and potential roles of CD1ddirected signaling in the regulation of mucosal inflammation.

\section{A novel mechanism for CD1d upregulation}

New and provocative insights into the regulation of CD1d expression are reported in this issue of the JCI by Colgan and colleagues (9). In this study, Colgan et al. examined whether CD1d expression could be regulated by intestinal luminal contents, using the colon-derived tissue culture cell lines T84, HT29, and CaCo-2 as model systems. These intestinal epithelioid cell lines express low levels of CD1d, in marked contrast to tissue IECs, which express CD1d in both the apical and the basolateral domains. However, when T84 cells were cultured in the presence of a soluble fraction derived from murine or human intestinal luminal contents, a significant increase in cell surface expression of CD1d was observed. Importantly, the relative expression of other cell surface proteins, i.e., MHC class II and ICAM-1, were not affected.

Experiments performed with luminal contents derived from germ/pathogen-free mice suggested that the factor or factors eliciting CD1d expression were not of microbial origin. Microsequencing analyses of the major protein components of a partially purified luminal content extract identified heat shock protein 110 (Hsp110), and, most remarkably, addition of purified recombinant Hsp110 to T84 monolayers recapitulated the effects of the luminal contents: recombinant Hsp110 elicited CD1d expression. Furthermore, Colgan et al. (9) report that Hsp110 is abundantly expressed in IECs, leading them to postulate that Hsp110 may undergo a regulated release from epithelia, where it would provide an autocrine signal for CD1d regulation.

\section{Hsp function in the extracellular space}

Hsp110 is a molecular chaperone that belongs to the Hsp100/Sse1 family of heat shock proteins, which are themselves distant relatives of the Hsp70 family of heat shock proteins $(10,11)$. Hsp110 is expressed in all mammalian tissues and is induced by heat shock. Like other heat shock proteins, increased expression of Hsp110 is associated with enhanced thermotolerance. Given its prominent role in intracellular protein folding and assembly, it is not immediately obvious why Hsp110 would function in an extracellular compartment to regulate cell surface CD1d expression. However, recent studies have demonstrated that heat shock/molecular chaperone proteins can function in an extracellular context to modulate and/or elicit innate and adaptive immune responses (12). In particular, many Hsp's have been reported to upregulate the expression of various cell surface proteins of dendritic cells (13).

\section{An autocrine function for Hsp110?}

As with all groundbreaking discoveries, the identification of Hsp110 as a potential autocrine inducer of $\mathrm{CD} 1 \mathrm{~d}$ expression raises many additional questions. To the cell biologist, the existence of substantial quantities of intact Hsp110 in intestinal luminal contents is something of a mystery. As Colgan et al. note, Hsp110 is not found in

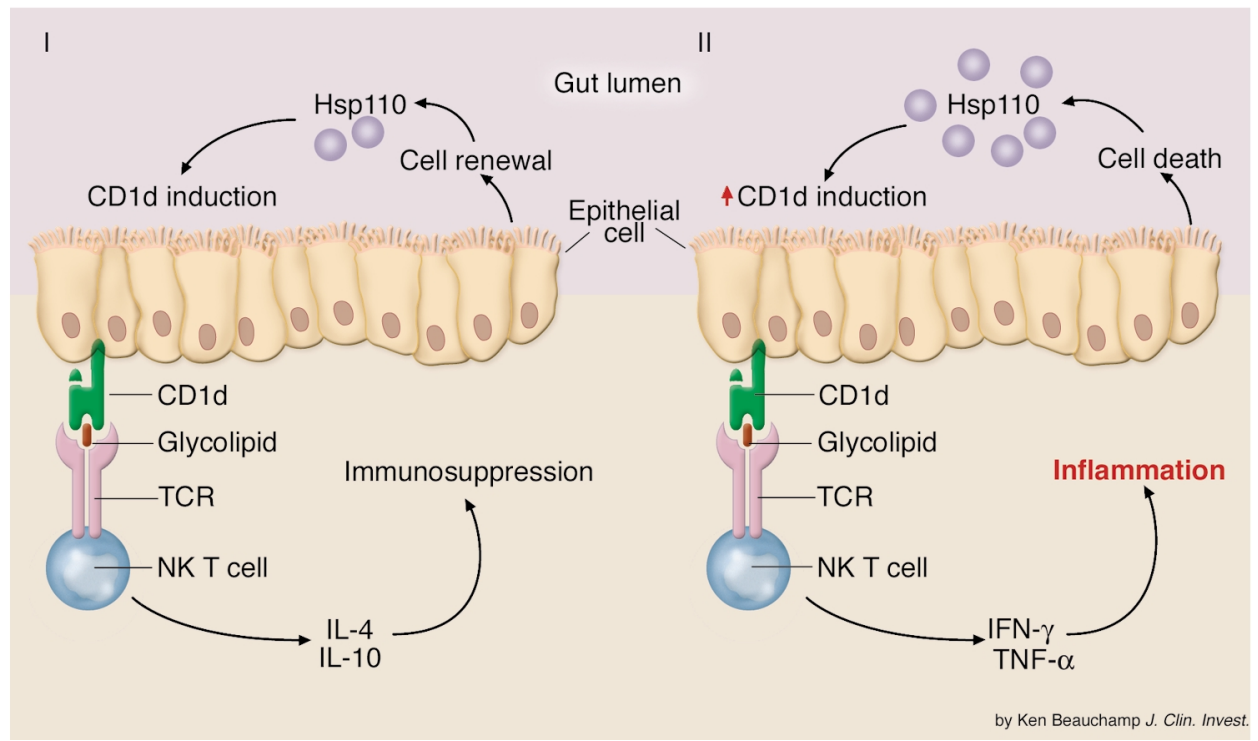

\section{Figure 1}

Hypothetical model for an Hsp110 function in IEC CD1d expression. (I) In this speculative model, Hsp110 release from IECs occurs during the physiological process of epithelial renewal and contributes, directly or indirectly, to the regulation of CD1d expression. In the case of normal (physiological) epithelial cell renewal, Hsp110 is released, whereupon it serves to signal normal (physiological) levels of CD1d expression. In this scenario, CD1d-directed NK T cell activation leads to the induction of IL-4 and IL-10 release and suppression of inflammatory responses. (II) In the case of accelerated (pathological) IEC death, Hsp110 release may be elevated, leading to enhanced levels of CD1d expression and perhaps alterations in the identity or relative quantity of CD1d ligands. In this scenario, NKT cells would be activated to yield release of the proinflammatory cytokines IFN- $\gamma$ and TNF- $\alpha$, thereby promoting the induction of inflammatory disease. TCR, T cell receptor. 
prokaryotes; therefore gut flora are not the source. A dietary source can be questioned, as it is unlikely that the Hsp110 would be recovered in a structurally intact form in the large intestine. And so the most likely source is the gut epithelium itself. Yet, Hsp110 does not possess a signal peptide and so cannot be secreted from IECs via the classical secretory pathway. Noncanonical secretion, as seen, for example, with FGF-2, is a possibility (14). However, this release pathway, and the analogous pathway in yeast, appear to transport very few proteins. Perhaps, then, Hsp110 is released into the intestinal lumen as a consequence of epithelial renewal, particularly in response to IEL activation (15). If this is the case, though, it might be expected that other abundant cytosolic chaperone proteins, such as Hsp90 and Hsp70, would be recovered in the intestinal contents as well. Nonetheless, perhaps inappropriately elevated levels of Hsp110 in the intestinal lumen serve as an immunological "trigger" leading to aberrant induction of CD1d expression, a subsequent activation of $\mathrm{NK} \mathrm{T}$ cells, and
IL-13-elicited destruction of IECs (Figure 1) (16). Whatever the mechanism, one is left to ponder the fascinating mystery of why IECs might (uniquely?) release Hsp110 into the gut lumen. Insights into the precise cellular source of the gut-lumen Hsp110 and the mechanism of its release will provide all-important clues to this new riddle in the everexpanding world of Hsp110 function.

1. Fiocchi, C. 1998. Inflammatory bowel disease: etiology and pathogenesis. Gastroenterology. 115:182-205

2. Blumberg, R.S. 2001. Characterization of CD1d in mucosal immune function: an immunotherapeutic target for inflammatory bowel disease. Keio J. Med. 50:39-44.

3. Mizoguchi, A., Mizoguchi, E., Takedatsu, H., Blumberg, R.S., and Bhan, A.K. 2002. Chronic intestinal inflammatory condition generates IL-10-producing regulatory B cell subset characterized by CD1d upregulation. Immunity. 16:219-230.

4. Page, M.J., et al. 2000. Cd1d-restricted cellular lysis by peripheral blood lymphocytes: relevance to the inflammatory bowel diseases. J. Surg. Res. 92:214-221.

5. Bendelac, A., et al. 1995. CD1 recognition by mouse NK1+ T lymphocytes. Science. 12:863-865.

6. Mendiratta, S.K., et al. 1997. CD1d1 mutant mice are deficient in natural $\mathrm{T}$ cells that promptly produce IL-4. Immunity. 6:467-477.

7. Colgan, S.P., Hershberg, R.M., Furuta, G.T., and Blumberg, R.S. 1999. Ligation of intestinal epithelial CD1d induces bioactive IL-10: critical role of the cytoplasmic tail in autocrine signaling. Proc. Natl.
Acad. Sci. U. S. A. 96:13938-13943.

8. Mowat, A.M., and Weiner, H.L. 1999. Oral tolerance: physiological basis and clinical applications. In Mucosal immunology. P.L. Ogra et al., editors. Academic Press Inc. San Diego, California, USA. 587-618.

9. Colgan, S.P., et al. 2003. Intestinal heat shock protein 110 regulates expression of CD1d on intestinal epithelial cells. J. Clin. Invest. 112:745-754. doi:10.1172/JCI200317241.

10. Lee-Yoon, D., Easton, D., Murawski, M., Burd, R. and Subjeck, J.R. 1995. Identification of a major subfamily of large hsp70-like proteins through the cloning of the mammalian $110-\mathrm{kDa}$ heat shock protein. J. Biol. Chem. 270:15725-15733.

11. Easton, D.P., Kaneko, Y., and Subjeck, J.R. 2000. The hsp110 and Grp1 70 stress proteins: newly recognized relatives of the Hsp70s. Cell Stress Chaperones. 5:276-290

12. Anderson, K.M., and Srivastava, P.K. 2000. Heat, heat shock, heat shock proteins and death: a central link in innate and adaptive immune responses. Immunol. Lett. 74:35-39.

13. Srivastava, P.K., and Amato, R.J. 2001. Hea shock proteins: the 'Swiss Army Knife' vaccines against cancers and infectious agents. Vaccine. 19:2590-2597.

14. Wakisaka, N., Murono, S., Yoshizaki, T. Furukawa, M., and Pagano, J.S. 2002. Epstein-Barr virus latent membrane protein 1 induces and causes release of fibroblast growth factor-2. Can cer Res. 62:6337-6344.

15. Guy-Grand, D., DiSanto, J.P., Henchoz, P., MalassisSeris, M., and Vassalli, P. 1998. Small bowel enteropathy: role of intraepithelial lymphocytes and of cytokines (IL-12, IFN-gamma, TNF) in the induc tion of epithelial cell death and renewal. Eur. J Immunol. 28:730-744.

16. Bouma, G., and Strober, W. 2003. The immunolog ical and genetic basis of inflammatory bowel disease. Nat. Rev. Immunol. 3:621-633.

\title{
Dendritic cells and the intestinal bacterial flora: a role for localized mucosal immune responses
}

\author{
Holm H. Uhlig and Fiona Powrie
}

Sir William Dunn School of Pathology, University of Oxford, Oxford, United Kingdom

Mammals coexist in an overall symbiotic relationship with a complex array of commensal bacterial flora that colonizes the gastrointestinal tract. These intestinal bacteria interface with cells of the mucosal immune system, including DCs (see the related article beginning on page 693). Here we discuss mechanisms of interaction between intestinal bacteria and DCs and the role of localized gastrointestinal immune responses.

J. Clin. Invest. 112:648-651 (2003). doi:10.1172/JCI200319545.

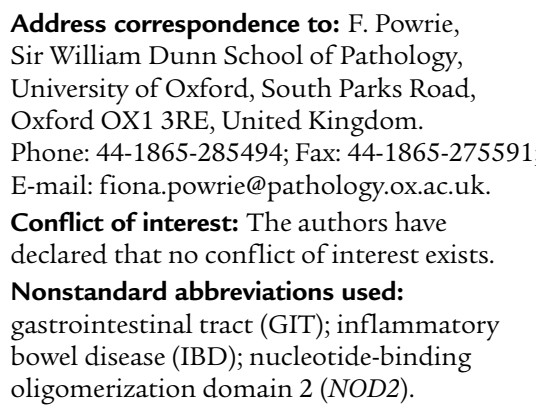

Inflammatory bowel disease is associated with a dysregulated immune response to intestinal bacterial flora

Through processes of evolutionary and individual adaptation, mammals coexist with an estimated 300 to 500 different species of commensal bacteria that colonize the gastrointestinal tract (GIT) in an overall symbiotic rela- tionship $(1,2)$. The presence of intestinal bacteria plays an important role in host metabolism, the development of the intestinal epithelium, and the intestinal immune system, and it also protects the host against rapid colonization by intestinal pathogens $(1,2)$. To allow sufficient defense against potential pathogens but restrict the immune response to nonpathogenic resident commensal bacteria, the mucosal immune system needs to be tightly regulated.

In human inflammatory bowel disease (IBD), which encompasses Crohn disease and ulcerative colitis, it is thought that a dysregulated $\mathrm{T}$ cell response to the intestinal bacterial microflora leads to chronic intestinal inflammation $(2,3)$. Although increasing evidence suggests that the intestinal flora is involved in the pathogenesis of human IBD, to date no specific bacterial pathogen has been identified. It seems more likely that different bacteria are involved in the initiation of the pathogenic immune response $(4,5)$. 